University of Nebraska - Lincoln

DigitalCommons@University of Nebraska - Lincoln

Faculty Publications - Chemistry Department Published Research - Department of Chemistry

2010

Concave Cubic Gold Nanocrystals with High-Index Facets

Jian Zhang

University of Nebraska-Lincoln, jzhang3@unl.edu

Mark R. Langille

Northwestern University, MarkLangille2013@u.northwestern.edu

Michelle L. Personick

Northwestern University

Ke Zhang

Northwestern University

Shuyou Li

Northwestern University

See next page for additional authors

Follow this and additional works at: https://digitalcommons.unl.edu/chemfacpub

Part of the Chemistry Commons

Zhang, Jian; Langille, Mark R.; Personick, Michelle L.; Zhang, Ke; Li, Shuyou; and Mirkin, Chad A., "Concave Cubic Gold Nanocrystals with High-Index Facets" (2010). Faculty Publications -- Chemistry Department. 52.

https://digitalcommons.unl.edu/chemfacpub/52

This Article is brought to you for free and open access by the Published Research - Department of Chemistry at DigitalCommons@University of Nebraska - Lincoln. It has been accepted for inclusion in Faculty Publications -Chemistry Department by an authorized administrator of DigitalCommons@University of Nebraska - Lincoln. 


\section{Authors}

Jian Zhang, Mark R. Langille, Michelle L. Personick, Ke Zhang, Shuyou Li, and Chad A. Mirkin 


\title{
Concave Cubic Gold Nanocrystals with High-Index Facets
}

\author{
Jian Zhang, ${ }^{1,3}$ Mark R. Langille, ${ }^{1,3}$ Michelle L. Personick, ${ }^{1,3}$ \\ Ke Zhang, ${ }^{1,3}$ Shuyou Li, ${ }^{2}$ and Chad A. Mirkin ${ }^{1,2,3}$
}

1. Department of Chemistry, 2. Department of Materials Science and Engineering, and 3. International Institute for Nanotechnology, Northwestern University, 2145 Sheridan Road, Evanston, Illinois 60208

The first two authors authors contributed equally.

Corresponding author - C. A. Mirkin, chadnano@northwestern.edu

\begin{abstract}
A new class of gold nanostructures, concave nanocubes, enclosed by 24 high-index $\{720\}$ facets, have been prepared in a monodisperse fashion by a modified seed-mediated synthetic method. The $\mathrm{Cl}^{-}$counterion in the surfactant plays an essential role in controlling the concave morphology of the final product. The concave nanocubes exhibit higher chemical activities compared with low-index $\{111\}$-faceted octahedra.
\end{abstract}

As the field of nanotechnology evolves, it is becoming clear that particle shape is as important as size in determining the chemical and physical properties of a particular nanostructure. This is especially true for noble metal nanoparticles, where the plasmonic and catalytic properties can be finely tuned through control over their morphology. ${ }^{1-7}$ Indeed, a variety of metallic nanostructures with well-defined shapes have been synthesized via photochemical, ${ }^{1,8-11}$ thermal, ${ }^{12-14}$ electrochemical, ${ }^{15}$ and template-directed methods. ${ }^{2,16}$ These synthetic strategies provide a means to chemically control the morphology of particles and, consequently, their catalytic, optical, electrical, and magnetic properties. ${ }^{4-6}$ In the case of catalysis, not only the shape of the particle but also the exposed crystalline faces are important, which can have a marked influence on both activity and selectivity. ${ }^{17,18}$ Interestingly, most synthetic methods to date yield nanostructures with convex shapes enclosed by lowindex $\{111\},\{100\}$, and/or $\{110\}$ facets, and there are very few methods ${ }^{19-22}$ that allow one to realize nanostructures with highindex faces. Herein, we describe how seed-mediated methods, originally developed by Murphy et al. ${ }^{23,24}$ and El-Sayed et al., ${ }^{25}$ can be modified with the appropriate surfactant to yield a novel class of high-index $\{720\}$-faceted Au concave nanocubes.

In a typical synthesis, Au seeds were prepared by reduction of $\mathrm{HAuCl}_{4}$ by $\mathrm{NaBH}_{4}$ in the presence of cetyltrimethylammonium chloride (CTAC) instead of the conventional cetyltrimethylammonium bromide (CTAB). A growth solution was prepared by consecutively adding $0.5 \mathrm{~mL}$ of $10 \mathrm{mM} \mathrm{HAuCl}_{4}$, $0.1 \mathrm{~mL}$ of $10 \mathrm{mM} \mathrm{AgNO}, 0.2 \mathrm{~mL}$ of $1.0 \mathrm{M} \mathrm{HCl}$, and then 0.1 $\mathrm{mL}$ of $100 \mathrm{mM}$ ascorbic acid (AA) to a $10 \mathrm{~mL}$ aqueous solution of 0.1 M CTAC. Concave Au nanocube synthesis was initiated by adding the seed particles to the growth solution (Supporting Information). The reaction was gently shaken immediately after the addition of the seeds and left undisturbed overnight. In general, the amount of seed particles added affects the size of the resulting concave nanocubes, with fewer seeds (i.e., fewer nucleation sites) leading to larger structures (vide infra).

The reaction products were extensively characterized by SEM and TEM (Figure 1). When the products lie flat on a substrate,

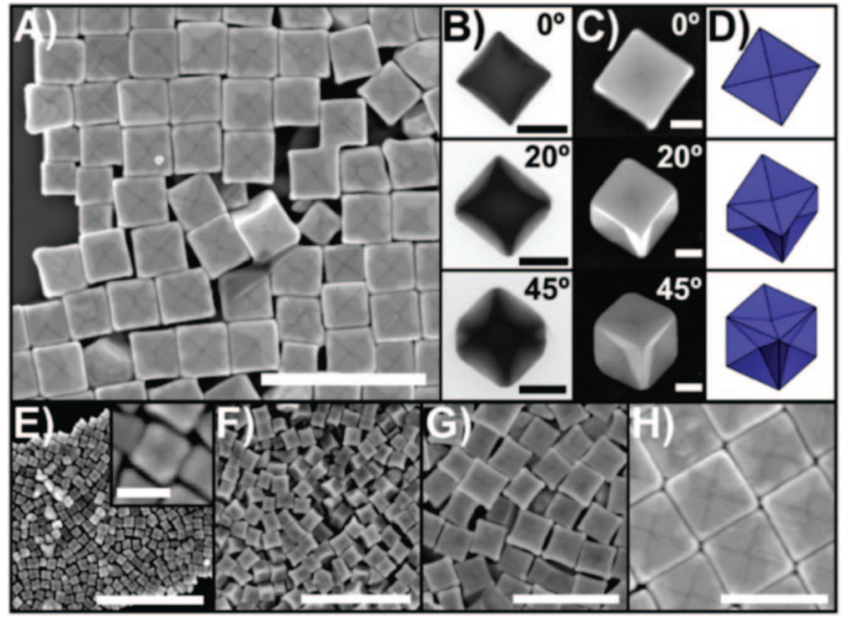

Figure 1. (A) Typical SEM image of Au concave cubes. Scale bar: 500 nm. (B) TEM, (C) SEM, and (D) model images of a concave cube tilted $0^{\circ}, 20^{\circ}$, and $45^{\circ}$ to illustrate the concave faces. Scale bars: $200 \mathrm{~nm}$. SEM images of concave cubes with edge lengths of (E) $38 \pm 7$, (F) $69 \pm 8$, (G) $115 \pm 19$, and $(\mathbf{H}) 269 \pm 18 \mathrm{~nm}$. Particle size was determined from $>100$ particles in each sample. Scale bars: $500 \mathrm{~nm}$. The inset of (E) shows a higher-magnification view, scale bar: $40 \mathrm{~nm}$.

the Au nanoparticles appear to have a cubic morphology. However, the SEM images (Figure 1A) show that the faces of the cubes each have contrast lines in the shape of an " $X$ ", and in the TEM images (Figure 1B) the cubes exhibit darker contrast in the middle region as compared with the edges. Incident beam angle-dependent TEM and SEM images show that each face of the cube is concave rather than flat (Figure $1 \mathrm{~B}$ and $1 \mathrm{C}$ ), and every particle inspected $(>1000)$ has these concave faces.

Remarkably, the size of the concave nanocubes, as determined by edge length, can be adjusted from tens to hundreds of nanometers by varying the volume of seed particles added to the growth solution. Therefore, concave cubes with edge lengths of $38 \pm 7,69 \pm 7,115 \pm 19$, or $269 \pm 18 \mathrm{~nm}$ can be synthesized (Figure 1E-H). SEM images of these products show that the concave faces of the cubes are maintained regardless of particle size, although the facets are better defined or more easily assignable for larger particles. A high yield (>95\%) of the concave cubes was observed for all nanocube sizes studied. The UV-vis spectroscopy of the smaller concave cubes (Figure S1) is consistent with the shape determined by TEM; the plasmon resonance is red-shifted by $80 \mathrm{~nm}$ compared to cubes with flat surfaces and of similar dimensions. ${ }^{26}$ Indeed, 


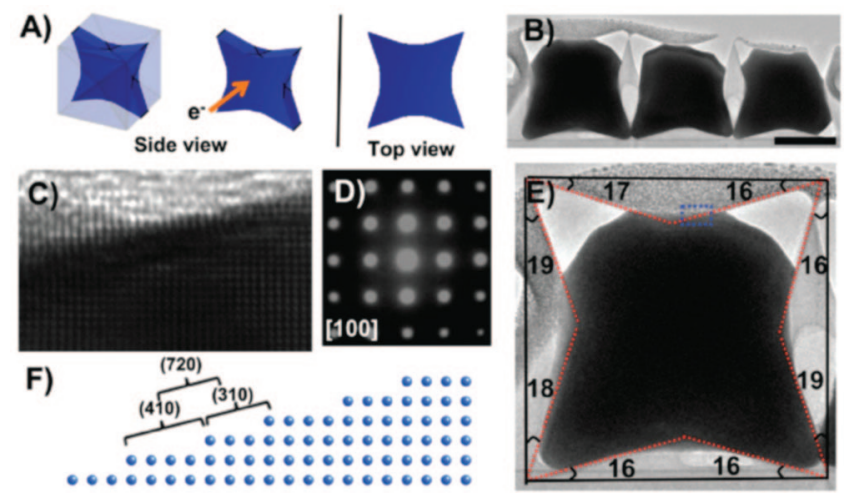

Figure 2. (A) Model and (B) TEM image of cross-sectioned concave cubes (scale bar: $200 \mathrm{~nm}$ ). (C) HRTEM image of the edge of the high-index facet. (D) Electron diffraction pattern of the sectioned concave cube in (C). (E) Zoomed-in image of a single cut concave cube aligned edgeon. (F) Atomic model of the $\{720\}$ surface, projected from the [001] direction, corresponding to the region indicated with a box in (E)

theory suggests that the plasmon resonance for the concave cubes, which have sharper tips than cubes with flat faces, is expected to be red-shifted. ${ }^{1}$

To fully characterize the novel structure of the concave nanocubes, it is important to index the surface facets. Due to the difficulty of directly imaging the concave faces, it was necessary to cut the crystal so that the concave facets can be imaged edge-on. To accomplish this difficult task, we utilized a focused ion beam (FIB) to cut the particle and expose a cross section of the concave cube along the [001] direction. The remaining section was then tilted to the [100] direction, as confirmed by electron diffraction (Figure 2D). Note that parts of the thin tips were lost during transfer and mounting of the sample for TEM analysis. The projected image fits well with the model (Figure 2A). The angles between the facets of the concave cube and the (100) facets of an ideal cube were determined to be $17^{\circ}, 16^{\circ}, 16^{\circ}, 19^{\circ}, 16^{\circ}$, $16^{\circ}, 18^{\circ}$, and $19^{\circ}$, respectively (Figure $2 \mathrm{E}$ ). Similar results were also obtained from the two other concave cubes. The average measured angle is $17 \pm 1^{\circ}$, indicating that the facets of the concave cubes are indeed high-index $\{720\}$ facets, which have a calculated angle of $16.0^{\circ}$. Note that some facets appear to have a slightly larger angle such as $18^{\circ}-19^{\circ}$, which can be attributed to a different high-index facet: $\{310\}$. Overall, we believe the facets of the concave cubes are mostly $\{720\}$ planes, mixed with some $\{310\}$ planes. A similar observation has also been made for the electrochemically prepared Pt tetrahexahedral nanoparticles, where, in addition to the most prevalent $\{730\}$ planes, a few other planes such as $\{520\},\{210\}$, and $\{310\}$ planes were also identified. ${ }^{19}$ The atomic arrangement of the $\{720\}$ planes was further confirmed using high-resolution TEM (HR-TEM) (Figure 2C). The $\{720\}$ facet is multiply stepped and composed of $\{410\}$ and $\{310\}$ subfacets (Figure $2 F$ ).

Nanostructures with concave faces are not common. ${ }^{21,27,28}$ The concave nanocubes reported here can be viewed as cubes with the centers of the six square faces "pushed in" to generate square pyramid-shaped depressions. This yields a nanostructure enclosed by 24 facets. It was recently reported that when $\mathrm{CTAB}$ rather than CTAC was used, tetrahexahedral (THH) $\mathrm{Au}$ nanoparticles were obtained even though the rest of the conditions were identical. ${ }^{22}$ Interestingly, the THH morphology can also be viewed as a cube with the centers of the six square faces "pulled out" to generate convex square pyramids (Fig-

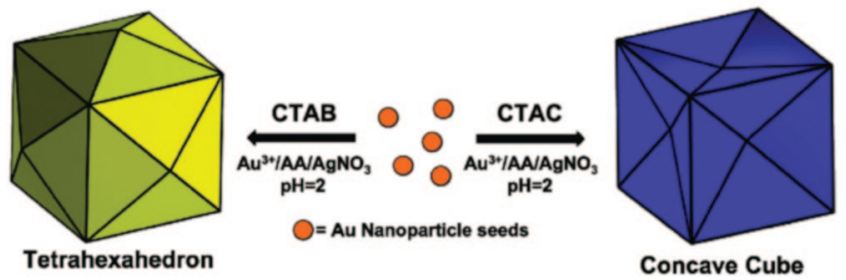

Figure 3. Schematic illustration showing the effect of the counterion on product morphology in the seed-mediated synthesis: CTAC leads to the formation of concave cubes, and CTAB leads to the formation of tetrahexahedra (convex cubes).

ure 3). We have discovered that a simple change of the halide counterion in the surfactant induces a dramatic change in the product morphology and yields a concave rather than convex cubic structure.

Although the formation mechanism is, as of now, unclear, we believe that it is the combination of $\mathrm{Ag}^{+}$and $\mathrm{Cl}^{-}$that gives rise to the concave cube morphology. $\mathrm{Ag}^{+}$has been used in other seed-mediated syntheses to stabilize high-index facets through an underpotential deposition (UPD) mechanism. ${ }^{29}$ Interestingly, it was found in correlated scanning tunneling microscopy/cyclic voltammetry experiments that the presence of $\mathrm{Cl}^{-}$significantly affects the UPD behavior of $\mathrm{Ag}^{+}$onto $\mathrm{Au}$, with $\mathrm{Ag}^{+}$being deposited specifically onto atomic steps below a $\mathrm{Cl}^{-}$adlayer, making the $\mathrm{Ag}^{+}$more difficult to remove. ${ }^{30,31}$ While both CTAB and CTAC have been used to synthesize nanostructures with high-index facets, ${ }^{21,22,29}$ CTAC has been attributed to the generation of nanoparticles with stellated features. ${ }^{21,32}$ Efforts aimed at understanding the role of the halide in the particle growth pathway are underway.

Concave Au cubes should have higher chemical activities than nanoparticle morphologies bound with low-index facets, due to the steps of the high-index $\{720\}$ facets. Cyclic voltammetry $(\mathrm{CV})$ is a method commonly used to study the surface structure of bulk $\mathrm{Au}^{33,34}$ and recently, this method has also been used to characterize the surface facets of Au nanostructures. ${ }^{21,22}$ The CV traces of the concave cubes with different sizes exhibit oxidation peaks around $1.2 \pm 0.5 \mathrm{~V}$ (Figure S2). This value is comparable to the result obtained for the THH Au nanoparticles enclosed by $\{730\}$ facets, which was measured to be 1.18 V. ${ }^{22}$ For comparison, the oxidation peak of octahedral Au nanoparticles (edge length $80 \mathrm{~nm}$ ) was determined to be $1.43 \mathrm{~V}$ (Figure S2). The oxidation peak, which is at a lower potential for the concave cubes than the octahedra, indicates that the surface atoms of the concave cubes are easier to oxidize. This is likely a consequence of the $\{720\}$ facets having a more open atomic arrangement as compared to the more tightly packed $\{111\}$ facets of the octahedra. This result clearly demonstrates higher activity for the higher-indexed concave cubes.

In conclusion, we have discovered a new class of $\mathrm{Au}$ nanoparticles, which have been characterized as concave nanocubes. These structures are related to their convex counterparts, tetrahexahedra, and are made simply by changing the counterion of the surfactant used in the seed-mediated synthesis. Extensive electron microscopy studies show that the concave nanocubes are single crystals enclosed by 24 high-index $\{720\}$ facets, and electrochemical measurements show that they are more chemically active (more susceptible to oxidation) than octahedral nanoparticles enclosed by low-index $\{111\}$ facets. Therefore, these structures may become useful candidates for fundamental structure versus function catalytic studies. 
Acknowledgment - This work was supported by the MRSEC program of the National Science Foundation at the Material Research Center of Northwestern University. The microscopy work was performed in the EPIC facility of NUANCE Center at Northwestern University. NUANCE Center is supported by NSF-NSEC, NSF-MRSEC, the Keck Foundation, the State of Illinois, and Northwestern University. We thank Ben Meyers for assistance with the FIB experiments. C.A.M. is also grateful for an NSSEF Fellowship from the DoD and support from the AFOSR.

\section{Supporting Information}

Experimental details, UV-vis extinction spectra, and cyclic voltammograms of different sized concave nanocubes and octahedra are presented following the References.

\section{References}

1. Jin, R. C., Cao, Y. W., Mirkin, C. A., Kelly, K. L., Schatz, G. C., and Zheng, J. G. Science 2001, 294, 1901

2. Hurst, S. J., Payne, E. K., Qin, L. D., and Mirkin, C. A. Angew. Chem., Int. Ed. 2006, 45, 2672

3. Millstone, J. E., Park, S. H., Shuford, K. L., Qin, L. D., Schatz, G. C., and Mirkin, C. A. J. Am. Chem. Soc. 2005, 127, 5312

4. Millstone, J. E., Hurst, S. J., Métraux, G. S., Cutler, J. I., and Mirkin, C. A. Small 2009, 5, 646

5. Xia, Y. N., Xiong, Y. J., Lim, B. K., and Skrabalak, S. E. Angew. Chem., Int. Ed. 2009, 48, 60

6. Tao, A. R., Habas, S., and Yang, P. D. Small 2008, 4, 310

7. Somorjai, G. A., Frei, H., and Park, J. Y. J. Am. Chem. Soc. 2009, 131, 16589

8. Maillard, M., Huang, P. R., and Brus, L. Nano Lett. 2003, 3, 1611

9. Jin, R. C., Cao, Y. C., Hao, E. C., Métraux, G. S., Schatz, G. C., and Mirkin, C. A. Nature 2003, 425, 487

10. Xue, C. and Mirkin, C. A. Angew. Chem., Int. Ed. 2007, 46, 2036

11. Zhang, J., Li, S. Z., Wu, J. S., Schatz, G. C., and Mirkin, C. A. Angew. Chem., Int. Ed. 2009, 48, 7787
12. Sun, Y. G. and Xia, Y. N. Science 2002, 298, 2176

13. Kim, F., Connor, S., Song, H. J., Kuykendall, T., and Yang, P. D. Angew. Chem., Int. Ed. 2004, 43, 3673

14. Wang, C., Daimon, H., Onodera, T., Koda, T., and Sun, S. H. Angew. Chem., Int. Ed. 2008, 47, 3588

15. Yu, Y. Y., Chang, S. S., Lee, C. L., and Wang, C. R. C. J. Phys. Chem. B 1997, 101, 6661

16. Martin, C. R. Science 1994, 266, 1961

17. Somorjai, G. A. and Blakely, D. W. Nature 1975, 258, 580

18. Sun, S. G., Chen, A. C., Huang, T. S., Li, J. B., and Tian, Z. W. J. Electroanal. Chem. 1992, 340, 213

19. Tian, N., Zhou, Z. Y., Sun, S. G., Ding, Y., and Wang, Z. L. Science 2007, 316, 732

20. Tian, N., Zhou, Z. Y., and Sun, S. G. J. Phys. Chem. C 2008, 112, 19801

21. Ma, Y. Y., Kuang, Q., Jiang, Z. Y., Xie, Z. X., Huang, R. B., and Zheng, L. S. Angew. Chem., Int. Ed. 2008, 47, 8901

22. Ming, T., Feng, W., Tang, Q., Wang, F., Sun, L. D., Wang, J. F., and Yan, C. H. J. Am. Chem. Soc. 2009, 131, 16350

23. Jana, N. R., Gearheart, L., and Murphy, C. J. J. Phys. Chem. B 2001, 105,4065

24. Sau, T. K. and Murphy, C. J. J. Am. Chem. Soc. 2004, 126, 8648

25. Nikoobakht, B. and El-Sayed, M. A. Chem. Mater. 2003, 15, 1957

26. Dovgolevsky, E. and Haick, H. Small 2008, 4, 2059

27. Zhou, Z. Y., Tian, N., Huang, Z. Z., Chen, D. J., and Sun, S. G. Faraday Discuss. 2008, 140, 81

28. Huang, X. Q., Tang, S. H., Zhang, H. H., Zhou, Z. Y., and Zheng, N. F. J. Am. Chem. Soc. 2009, 131, 13916

29. Liu, M. Z. and Guyot-Sionnest, P. J. Phys. Chem. B 2005, 109, 22192

30. Lee, J. H., Oh, I. W., Hwang, S. P., and Kwak, J. Y. Langmuir 2002, 18,8025

31. Michalitsch, R., Palmer, B. J., and Laibinis, P. E. Langmuir 2000, 16, 6533

32. Wu, H. L., Chen, C. H., and Huang, M. H. Chem. Mater. 2009, 21, 110

33. Hamelin, A. J. Electroanal. Chem. 1996, 407, 1

34. Hamelin, A. and Martins, A. M. J. Electroanal. Chem. 1996, 407, 13 


\title{
Supporting Information for
}

\section{Concave Cubic Gold Nanocrystals with High-Index Facets}

\author{
Jian Zhang, Mark R. Langille, Michelle L. Personick, Ke Zhang, Shuyou Li, and Chad A. Mirkin*
}

Department of Chemistry, Department of Materials Science and Engineering, and International Institute for Nanotechnology, Northwestern University, 2145 Sheridan Road, Evanston, IL 60208

E-mail: chadnano@northwestern.edu

\section{Experimental Details}

Au seeds were prepared by quickly injecting $0.60 \mathrm{~mL}$ of ice-cold, freshly prepared $\mathrm{NaBH}_{4}(10 \mathrm{mM})$ into a rapidly stirred solution containing $0.25 \mathrm{~mL}$ of $\mathrm{HAuCl}_{4}(10 \mathrm{mM})$ and $10.00 \mathrm{~mL}$ of cetyltrimethylammonium chloride (CTAC) $(100 \mathrm{mM})$. The seed solution was stirred for 1 minute and then left undisturbed for 2 hours. A growth solution was prepared by consecutively adding $0.50 \mathrm{~mL}$ of $\mathrm{HAuCl}_{4}(10 \mathrm{mM}), 0.10 \mathrm{~mL}$ of $\mathrm{AgNO}_{3}(10 \mathrm{mM}), 0.20 \mathrm{~mL}$ of $\mathrm{HCl}(1.0 \mathrm{M})$, then $0.10 \mathrm{~mL}$ of ascorbic acid $(100 \mathrm{mM})$ into $10.00 \mathrm{~mL}$ of $100 \mathrm{mM} \mathrm{CTAC}$. The seed particles were serially diluted in $0.1 \mathrm{M} \mathrm{CTAC}$ to generate solutions ranging from $1 / 10$ to $1 / 10^{4}$ the concentration of the original seed solution. A typical synthesis of concave cubes was initiated by the addition of $0.1 \mathrm{~mL}$ of the diluted seeds to the growth solution. The reaction was swirled immediately after the addition of the seeds and then left undisturbed on the bench top until the reaction completed.

SEM and TEM characterizations were conducted on a Hitachi S-4800-II cFEG SEM and a JEOL JEM-2100F FEG FasTEM, respectively. The concave nanocubes were sectioned and mounted on a TEM grid using the "H-bar lift-out" technique " on a FEI Helios Nanolab 600 FIB instrument.

The CV measurements were carried out in a three-electrode cell using a BAS 100B/W Electrochemical Analyzer. The Au nanocrystals were washed twice by centrifugation and then drop-cast onto indium tin oxide substrates. The substrates with the deposited Au nanocrystals were washed again with ethanol and then used as the working electrodes. A Pt wire and $\mathrm{Ag} / \mathrm{AgCl}$ electrode (in saturated $\mathrm{NaCl}$ ) served as the counter and reference electrodes, respectively. An aqueous solution of $\mathrm{H}_{2} \mathrm{SO}_{4}(0.1 \mathrm{M})$ was employed as the electrolyte solution. The electrochemical set-up was purged with high-purity $\mathrm{N}_{2}$ gas for 30 min prior to measurements. The voltage scan rate was $20 \mathrm{mVs}^{-1}$. The first two cycles of oxidation and reduction were discarded due to the presence of adsorbed gas molecules. The data recorded during the third cycle are shown here. The oxidation and reduction traces have been normalized against the maximum reduction current for each sample.

\section{References}

(1) Li, J.; Malis, T.; Dionne, S. Mater. Charact. 2006, 57, 64. 


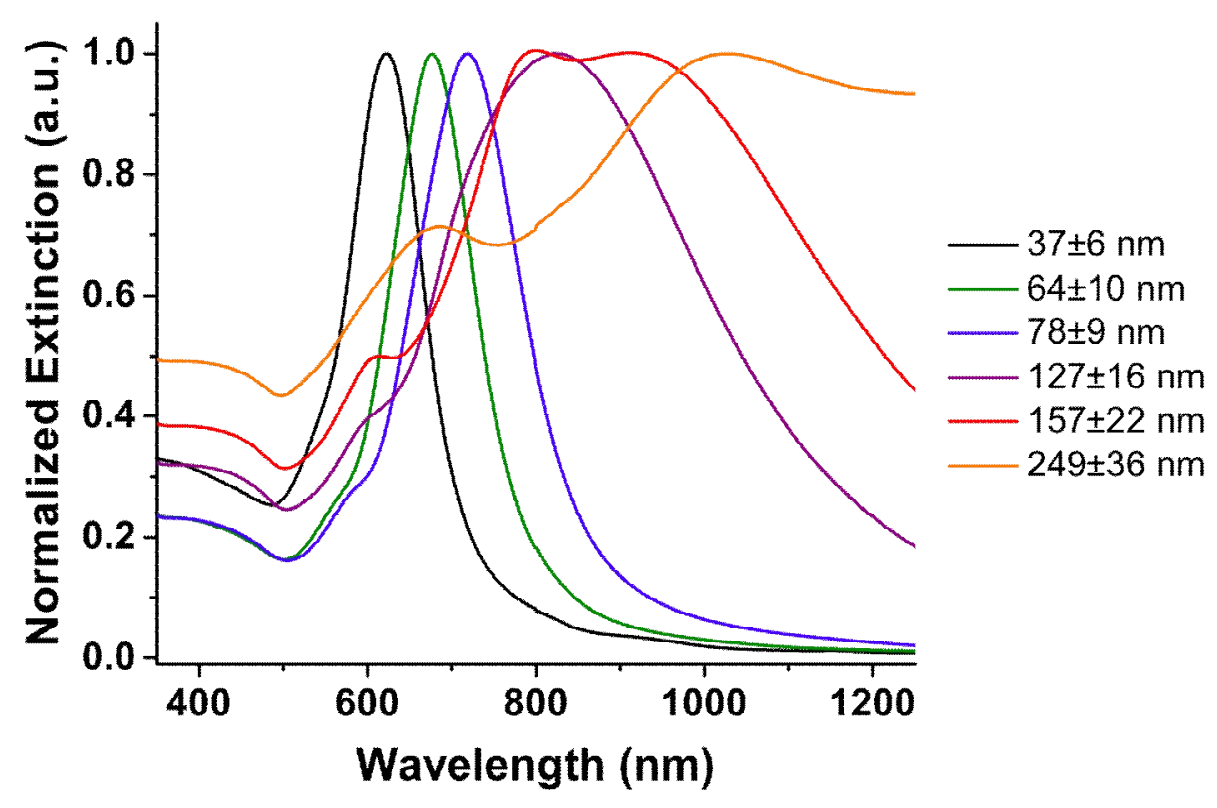

Figure S1. Normalized extinction spectra of the gold concave nanocubes with different edge lengths. The concave nanocubes with edge lengths of $37 \pm 6,64 \pm 10,78 \pm 9,127 \pm 16,157 \pm 22$, and $249 \pm 36 \mathrm{~nm}$ were synthesized by adding the equivalent of $5,1,0.5,0.1,0.05$, and $0.01 \mu \mathrm{L}$ of the non-diluted seed solution, respectively. 

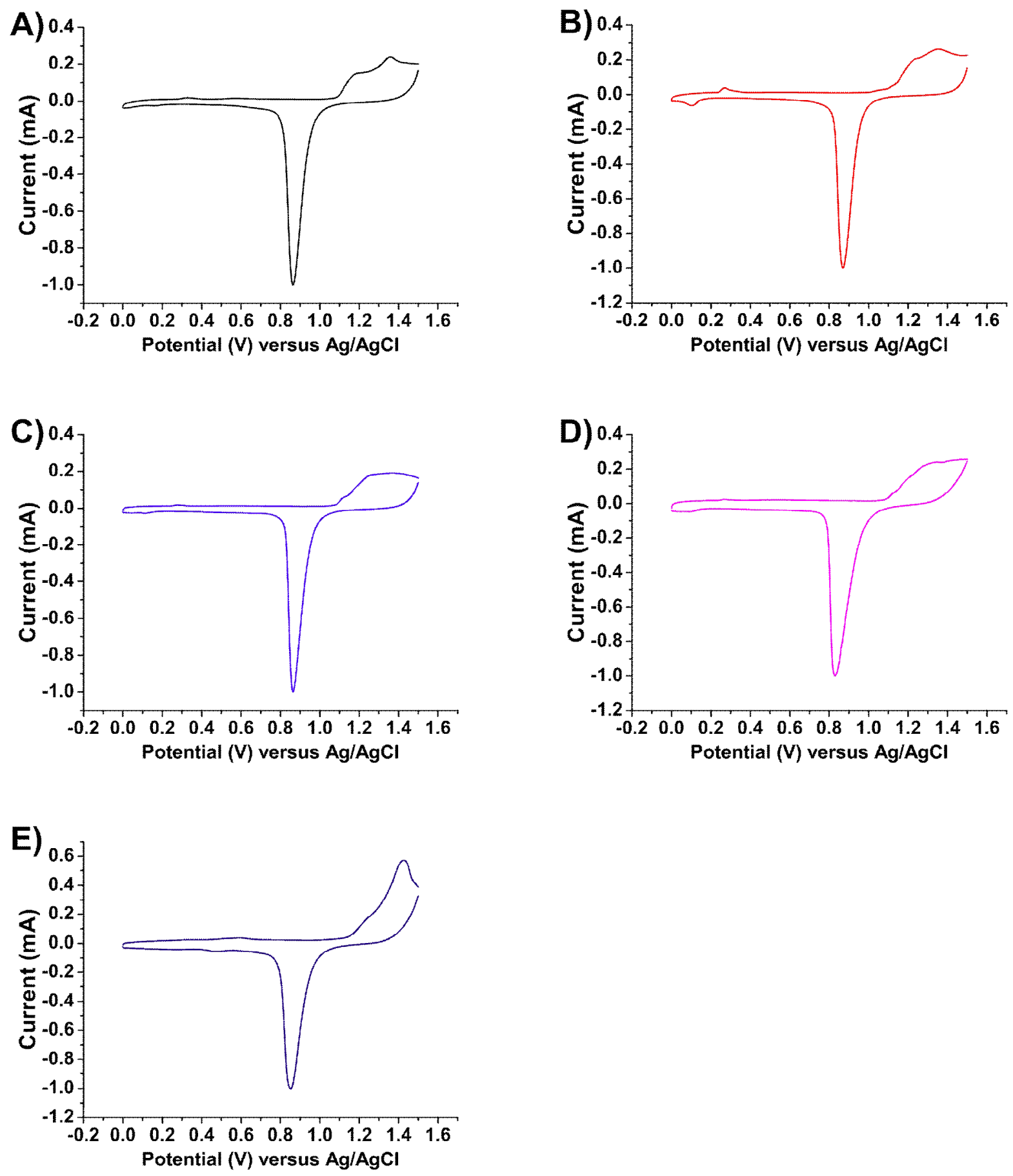

Figure S2. Cyclic voltammograms of Au concave cubes (A-D) with edge lengths of A) 38 \pm 7 , B) $69 \pm 8$, C) $115 \pm 19, \mathrm{D}) 269 \pm 18 \mathrm{~nm}$, and Au octahedra (E) (edgelength $120 \mathrm{~nm}$ ) at $10 \mathrm{mV} \mathrm{S}^{-1}$ in $0.1 \mathrm{M} \mathrm{H}_{2} \mathrm{SO}_{4}$. 